\title{
Patient-Reported Outcomes and Quality of Life after Treatment for Choroidal Melanoma
}

\author{
Bertil Damato $^{\mathrm{a}, \mathrm{b}}$ Laura Hope-Stone ${ }^{\mathrm{b}}$ Bruce Cooper $^{\mathrm{a}}$ Steve Brown $^{\mathrm{b}}$ \\ Heinrich Heimann ${ }^{b}$ Laura Dunn ${ }^{c}$

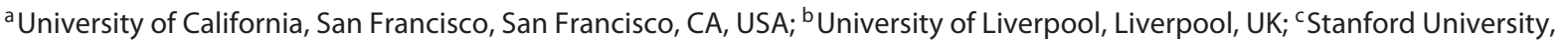 \\ Stanford, CA, USA
}

\section{Keywords}

Choroidal melanoma · Enucleation · Radiotherapy · Quality of life $\cdot$ Anxiety $\cdot$ Depression $\cdot$ Well-being

\begin{abstract}
Background/Aims: Patients with choroidal melanoma can develop psychological morbidity because of visual disability, pain, facial deformity, and fears of metastatic disease. The aim of this study was to report on the prevalence of symptoms, moods, and well-being after radiotherapy or enucleation for choroidal melanoma and how these outcomes changed over time. Methods: Participants were mailed questionnaires approximately 6 months following treatment, then annually on every anniversary of their treatment. $\boldsymbol{R e}$ sults: Soon after enucleation, patients experienced visual difficulties because of loss of stereopsis and visual field and were concerned about their appearance and about metastatic disease. After radiotherapy, patients were more concerned about local tumor recurrence and more troubled by diplopia and headache. Over time, visual difficulties diminished after enucleation but increased in patients who had received radiotherapy, with concerns about metastasis, loss of health, and tumor recurrence diminishing in both groups.
\end{abstract}

Anxiety tended to diminish whereas depression increased, especially after enucleation. Emotional well-being improved after both kinds of treatment, whereas functional and physical well-being diminished after enucleation but improved after radiotherapy. Self-reported quality of life diminished equally with both kinds of treatment. Conclusion: The findings of this study should help physicians understand what patients tend to feel after treatment for choroidal melanoma.

(c) 2019 S. Karger AG, Basel

Patients with choroidal melanoma can develop visual disability, pain, facial deformity, and metastatic disease. These problems or the threat of such outcomes can cause anxiety, depression, and loss of well-being.

Physicians and other healthcare providers need to know of the psychological difficulties that patients might develop so as to be able to prevent and treat such problems. Such awareness is also useful when helping patients select treatment.

For many years, enucleation was the standard treatment for choroidal melanoma. When the Collaborative Ocular Melanoma Study concluded that radiotherapy was as effective at "prolonging life," it became standard practice to con-

\section{KARGER}

(C) 2019 S. Karger AG, Basel

E-Mail karger@karger.com

www.karger.com/oop
Dr. Bertil Damato, MD, PhD, FRCOphth

Nuffield Department of Clinical Neurosciences

University of Oxford, West Wing, John Radcliffe Hospital

Oxford OX3 9DU (UK)

E-Mail Bertil.Damato@NHS.net 
serve the eye whenever possible, in the hope of preserving quality of life (QoL) [1].

Several studies have compared enucleation with radiotherapy with respect to QoL, with conflicting results [1-5]. In a previous study, we concluded that although QoL is worse after enucleation, this is mostly because of factors predisposing to delayed presentation and the need for enucleation, rather than the treatment itself [6]. Patients who underwent enucleation tended to be older, with a more advanced tumor, a higher risk of metastasis, unrelated illness and other problems, which contributed to loss of well-being. In fact, of those reporting poor QoL, only $20 \%$ indicated that this was caused by their ocular condition [6].

In our previous study, many patients completed several questionnaires over several years; however, we analyzed only the first questionnaire submitted, without taking time into account [6]. This may have biased the results, because enucleated patients had a shorter follow-up than those who had received radiotherapy.

In this study, we analyzed all submitted questionnaires to report how symptoms, moods, and well-being changed over time after radiotherapy or enucleation for choroidal melanoma.

\section{Patients and Methods}

Our methods have been described in detail [6]. Briefly, the study sample comprised a consecutive series of patients who resided in England or Wales, who were treated for choroidal melanoma at the Liverpool Ocular Oncology Centre (LOOC) between January 1993 and December 2013, and who consented to participate in this study. Melanomas were diagnosed according to generally accepted clinical features or by biopsy.

Treatment was selected according to ocular findings and the patient's needs and fears. Survival prognostication was based on clinical, histologic, and genetic predictors, using an online tool developed at LOOC [7]. Patients received an audio-recording of their consultation to remember what was said, in addition to printed and online information [8]. Psychological support was provided by specialist nurses and by a full-time health psychologist working exclusively for LOOC.

Participants were mailed questionnaires approximately 6 months following treatment, then annually on every anniversary of their treatment.

Patient-reported outcomes were measured using the European Organization for Research and Treatment of Cancer (EORTC) QLQ-OPT30 instrument [6, 9]. This comprises 27 questions each measuring the extent of a symptom during the previous week, which was scored from 1 to 4 as "not at all," "a little," "quite a bit," and "very much." The instrument has 7 questions assessing discomfort, 13 assessing visual difficulties, and 6 assessing worries about appearance and health (see Table 3a). The first author devised additional questions asking patients to rate their QoL and overall general health from 1 to 7 , with higher values indicating better outcomes. Patients were also asked to score from 1 to 7 the extent to which their QoL and general health were affected by their ocular condition, with higher scores indicating that these were more strongly affected by their ocular disease [6]. Anxiety and depression were measured using the Hospital Anxiety \& Depression Scale (HADS), which poses 7 questions assessing anxiety symptoms and 7 questions assessing depressive symptoms [10]. Each item is rated on a scale of $0-3$. Scores from 0 to 7,8 to 10 , and 11 to 21 are considered to indicate "normal," "borderline," and "abnormal/severe" levels of anxiety or depression, respectively. Physical, functional, emotional, and social well-being were measured using the Functional Assessment of Cancer Therapy scale (FACT-G) [11, 12]. Each symptom was scored as "not at all," "a little bit," "somewhat," "quite a bit," and "very much." Higher scores indicate better well-being. Mean scores for each of these four aspects of QoL have been published for the adult general and cancer populations in the United States, with scores exceeding 0.5 standard deviation below the mean for each subscale indicating low well-being in that specific domain [13]. We emphasize that higher scores indicate worse outcomes with the EORTC and HADS instruments but better outcomes with the FACT-G instrument and self-reported QoL.

Clinical and QoL data were computerized prospectively using a registry (Revelation Software, Westwood, NJ, USA), customized for LOOC by Sprezzatura (Sprezzatura Ltd, London, UK). Statistical analyses were performed with Stata/IC 14.1 (StataCorp, Texas, USA) and IBM SPSS Statistics, Version 22 (IBM Corp, New York, NY, USA). Between-group differences for categorical variables were examined with the Pearson $\chi^{2}$ test of independence, and with ordinal logistic regression for ordinal categorical data. Continuous variables were analyzed with the two-sample $t$ test with unequal variance, using the bootstrap (with 1,000 replications) for skewed variables.

Multilevel linear regression models were used to test linear change across time for anxiety, depression, self-reported QoL, and FACT-G scores. When patients completed more than one questionnaire in a time period, only the first questionnaire during that period was employed in the analyses. For non-normal distributions of outcome measures, we used the nonparametric bootstrap to obtain nonparametric, bias-corrected, bootstrapped, confidence intervals using 1,000 repetitions.

\section{Results}

\section{Demographics}

The cohort consisted of 442 enucleated patients and 1,154 patients who had received radiotherapy, which consisted of ruthenium plaque brachytherapy in $730 \mathrm{pa}-$ tients and proton beam radiotherapy in 424 (Table 1). Compared to those who had radiotherapy, enucleated patients were older, male, with poorer visual acuity in the affected eye, and with a larger, more anterior tumor, which had a higher prevalence of extraocular spread, epithelioid cells and chromosome 3 loss. Statistical analysis was performed on data from 1,615 questionnaires completed by enucleated patients and 4,619 questionnaires from patients who had received radiotherapy (Table 2). 
Table 1. Comparison between irradiated and enucleated patients

a Categorical variables

\begin{tabular}{|c|c|c|c|c|}
\hline Variable & $n$ & Enucleation, $n(\%)$ & Radiotherapy, $n(\%)$ & Statistics \\
\hline \multicolumn{5}{|l|}{ Sex } \\
\hline Female & 771 & $188(42.5)$ & $583(50.5)$ & \multirow{2}{*}{$\chi^{2}=8.16, p=0.004$} \\
\hline Male & 825 & $254(57.5)$ & $571(49.5)$ & \\
\hline \multicolumn{5}{|l|}{ Tumor-affected eye } \\
\hline Left & 790 & $228(51.6)$ & $562(48.7)$ & \multirow{2}{*}{$\chi^{2}=1.063, p=0.303$} \\
\hline Right & 806 & $214(48.4)$ & $592(51.3)$ & \\
\hline \multicolumn{5}{|l|}{ Visual acuity } \\
\hline $20 / 15-20 / 40$ & 1,106 & $171(38.7)$ & $935(81)$ & \multirow[t]{4}{*}{$\chi^{2}=292.65, p<0.001$} \\
\hline $20 / 60-20 / 200$ & 322 & $148(33.5)$ & $174(15.1)$ & \\
\hline $20 / 400-$ count fingers & 92 & $56(12.7)$ & $36(3.1)$ & \\
\hline Hand motions - no light perception & 76 & $67(15.2)$ & $9(0.8)$ & \\
\hline \multicolumn{5}{|l|}{ Anterior tumor margin } \\
\hline Post-equatorial choroid & 595 & $102(23.1)$ & $493(42.8)$ & \multirow{4}{*}{$\chi^{2}=101.25, p<0.001$} \\
\hline Pre-equatorial choroid & 645 & $170(38.6)$ & $475(41.2)$ & \\
\hline Ciliary body & 271 & $120(27.2)$ & $151(13.1)$ & \\
\hline Anterior chamber & 83 & $49(11.1)$ & $34(3)$ & \\
\hline \multicolumn{5}{|l|}{ Posterior tumor margin } \\
\hline Pre-equatorial uvea & 200 & $41(9.3)$ & $159(13.8)$ & \multirow[t]{5}{*}{$\chi^{2}=57.16, p<0.001$} \\
\hline Post-equatorial uvea & 689 & $169(38.2)$ & $520(45.1)$ & \\
\hline 1-2 disc diameters from disc/fovea & 187 & $28(6.3)$ & $159(13.8)$ & \\
\hline$<1$ disc diameters from disc/fovea & 309 & $70(15.8)$ & $239(20.7)$ & \\
\hline Involving disc & 211 & $134(30.3)$ & $77(6.7)$ & \\
\hline \multicolumn{5}{|l|}{ Coronal tumor location } \\
\hline Nasal & 582 & $225(50.9)$ & $357(30.9)$ & \multirow{3}{*}{$\chi^{2}=68.38, p<0.001$} \\
\hline Midline & 318 & $92(20.8)$ & $226(19.6)$ & \\
\hline Temporal & 696 & $125(28.3)$ & $571(49.5)$ & \\
\hline \multicolumn{5}{|l|}{ Sagittal tumor location } \\
\hline Superior & 572 & $143(32.4)$ & $114(25.8)$ & \multirow{3}{*}{$\chi^{2}=2.26, p=0.133$} \\
\hline Horizontal & 386 & $114(25.8)$ & $272(23.6)$ & \\
\hline Inferior & 638 & $185(41.9)$ & $453(39.3)$ & \\
\hline \multicolumn{5}{|l|}{ Extraocular tumor spread } \\
\hline No & 1,520 & $379(85.8)$ & $1,141(98.9)$ & \multirow[t]{2}{*}{$\chi^{2}=121.4302, p<0.001$} \\
\hline Yes & 76 & $63(14.3)$ & $13(1.1)$ & \\
\hline \multicolumn{5}{|l|}{ Epithelioid cytomorphology } \\
\hline No & 371 & $165(37.6)$ & $206(58.5)$ & \multirow{2}{*}{$\chi^{2}=34.3876, p<0.001$} \\
\hline Yes & 420 & $274(62.4)$ & $146(41.5)$ & \\
\hline \multicolumn{5}{|l|}{ Chromosome 3 status } \\
\hline Disomy 3 & 322 & $169(43.4)$ & $153(59.8)$ & \multirow[t]{2}{*}{$\chi^{2}=16.4505, p<0.001$} \\
\hline Monosomy 3 & 323 & $220(56.6)$ & $103(40.2)$ & \\
\hline
\end{tabular}

b Continuous variables

\begin{tabular}{|c|c|c|c|c|c|c|c|c|c|c|c|c|c|}
\hline \multirow[t]{3}{*}{ Variable } & \multicolumn{4}{|c|}{ Enucleation } & \multicolumn{4}{|c|}{ Radiotherapy } & \multicolumn{4}{|c|}{ Difference } & \multirow[t]{3}{*}{ Statistics } \\
\hline & \multirow[t]{2}{*}{$n$} & \multirow[t]{2}{*}{$\mathrm{Mn}$} & \multirow[t]{2}{*}{ Med } & \multirow[t]{2}{*}{ SD } & \multirow[t]{2}{*}{$n$} & \multirow[t]{2}{*}{$\mathrm{Mn}$} & \multirow[t]{2}{*}{ Med } & \multirow[t]{2}{*}{ SD } & \multirow[t]{2}{*}{$\mathrm{Mn}$} & \multirow[t]{2}{*}{ SD } & \multicolumn{2}{|l|}{$95 \% \mathrm{CI}$} & \\
\hline & & & & & & & & & & & lower & upper & \\
\hline Age, years & 442 & 0.56 & 64.63 & 11.78 & 1,154 & 60.99 & 61.54 & 13.04 & 3.33 & 0.68 & 1.99 & 4.66 & $t(877.913)=4.898 ; p<0.001$ \\
\hline Basal tumor diameter, mm & 439 & 15.08 & 15.30 & 3.33 & 1,150 & 10.99 & 10.80 & 2.99 & 4.08 & 0.18 & 3.73 & 4.44 & $t(724.294)=22.481 ; p<0.001$ \\
\hline Tumor thickness, mm & 440 & 7.74 & 7.90 & 3.46 & 1,154 & 3.31 & 2.80 & 1.89 & 4.42 & 0.18 & 4.07 & 4.79 & Bias-corrected bootstrap \\
\hline Follow-up, months & 442 & 61.09 & 37.91 & 51.95 & 1154 & 76.61 & 60.04 & 60.56 & -15.52 & 3.05 & -21.50 & -9.54 & $t(923.834)=-5.094 ; p<0.001$ \\
\hline
\end{tabular}

Mn, mean; Med, median; SD, standard deviation; SE, standard error; CI, confidence interval. 


\section{Baseline Data}

Data from the EORTC QLQ-OPT-30 questionnaires indicated that the most troublesome difficulties after enucleation were: worries about metastasis (mean, 2.690), losing the eye (2.457), and future health (mean, 2.410); difficulty judging distance (mean, 2.291), walking in crowds (mean, 2.192), and seeing steps (mean, 2.143) (Table 3a; Fig. 1, 2).

Compared to enucleated patients, those who had received radiotherapy were more likely to report: fears of local tumor recurrence (obs. coeff., 0.291); headache (obs. coeff., 0.106); and diplopia when looking to side (obs. coeff., 0.368); and less likely to experience: worries about metastasis (obs. coeff., -0.164); loss of future health (obs. coeff., -0.113); visual difficulties, such as walking in crowds (obs. coeff., -0.848), pouring drinks (obs. coeff., -0.758 ); and ocular discomforts, such as ocular discharge (obs. coeff., -0.665); and worries about appearance (obs. coeff., -0.387 ). Data from the HADS and FACT-G questionnaires indicated that patients who had radiotherapy were less likely to suffer from depression (obs. coeff. -0.408) and loss of emotional well-being (obs. coeff., 0.518).

\section{Change over Time}

After enucleation, EORTC QLQ-OPT30 data indicated that the following outcomes decreased in a linear fashion over time: concerns about local tumor recurrence (obs. coeff., -0.111), loss of future health (obs. coeff., -0.0115 ), metastatic disease (obs. coeff., -0.141 ), and appearance (obs. coeff., -0.058 ); difficulty walking in crowds (obs. coeff., - 0.018 ) and pouring drinks (obs. coeff., -0.025 ); discomfort from watering (obs. coeff., 0.026) and a foreign body sensation (obs. coeff., -0.035). HADS and FACT-G data indicated that anxiety diminished (obs. coeff., -0.189) and emotional well-being improved (obs. coeff., 0.181) (Table 3b). Conversely, functional well-being (obs. coeff., -0.177) and physical well-being (obs. coeff., -0.102 ); and self-reported QoL (obs. coeff., -0.029) declined, whereas depression increased (obs. coeff., 0.05).

Several changes over time showed quadratic trends, mostly worsening significantly after an initial improvement. These include: grittiness (obs. coeff., linear: -0.035 ; quadrantic: 0.003); visual field loss (obs. coeff., linear: -0.138 ; quadratic: -0.011$)$; and worries about appearance (obs. coeff., linear: -0.058; quadratic: 0.005), local recurrence (obs. coeff., linear: -0.111 ; quadratic: 0.006 ), losing eye (obs. coeff., linear: -0.126 ; quadratic: 0.007 ), future health (obs. coeff., linear: -0.115 ; quadratic: 0.007 ), and metastasis (obs. coeff., linear: -0.141 ; quadratic: 0.008 ). There was also a late increase in anxiety (obs. coeff., lin-
Table 2. Number of questionnaires completed per patient and per follow-up time after enucleation or radiotherapy

a Questionnaires completed per patient

\begin{tabular}{lccr}
\hline Questionnaires & \multicolumn{2}{c}{ Treatment } & \multirow{2}{*}{ Total } \\
\cline { 2 - 3 } & enucleation & radiotherapy & \\
\hline 1 & 426 & 1,112 & 1,538 \\
2 & 350 & 968 & 1,318 \\
3 & 255 & 766 & 1,021 \\
4 & 199 & 608 & 807 \\
5 & 142 & 452 & 594 \\
6 & 106 & 315 & 421 \\
7 & 83 & 227 & 310 \\
8 & 39 & 132 & 171 \\
9 & 15 & 39 & 54 \\
\hline Total & 1,615 & 4,619 & 6,234 \\
\hline
\end{tabular}

b Questionnaires completed per follow-up time period

\begin{tabular}{lccc}
\hline Follow-up & \multicolumn{2}{l}{ Treatment } & \multirow{2}{*}{ Total } \\
\cline { 2 - 3 } & enucleation & radiotherapy & \\
\hline 0.5 years & 185 & 422 & 607 \\
1 year & 236 & 516 & 752 \\
2 years & 196 & 469 & 665 \\
3 years & 155 & 404 & 559 \\
4 years & 128 & 376 & 504 \\
5 years & 112 & 342 & 454 \\
6 years & 108 & 300 & 408 \\
7-8 years & 192 & 553 & 745 \\
9-10 years & 136 & 417 & 553 \\
$>10$ years & 167 & 820 & 687 \\
\hline Total & 1,615 & 4,619 & 6,234 \\
\hline
\end{tabular}

ear: -0.189 ; quadratic: 0.013$)$ with loss of emotional wellbeing (obs. coeff., linear: -0.181 ; quadratic: -0.011 ). These appear as curved lines in Figures 1, 2.

Differences in outcomes between the two kinds of treatment diminished with respect to visual difficulties such as walking in crowds (obs. coeff., 0.101) and pouring drinks (obs. coeff., 0.043), because of improvement following enucleation and deterioration after radiotherapy. The overall FACT-G scores diverged (obs. coeff., 0.450) as functional (obs. coeff., 0.181) and physical (obs. coeff., 0.090) well-being diminished after enucleation but improved after radiotherapy. Visual field loss, diplopia looking to side and worries about appearance showed a variety of combinations of linear and quadratic trends (Fig. 1,2). 
Table 3. Baseline patient-reported outcome measures, moods and QoL, and trends

a Baseline patient-reported outcome measures, moods, and QoL

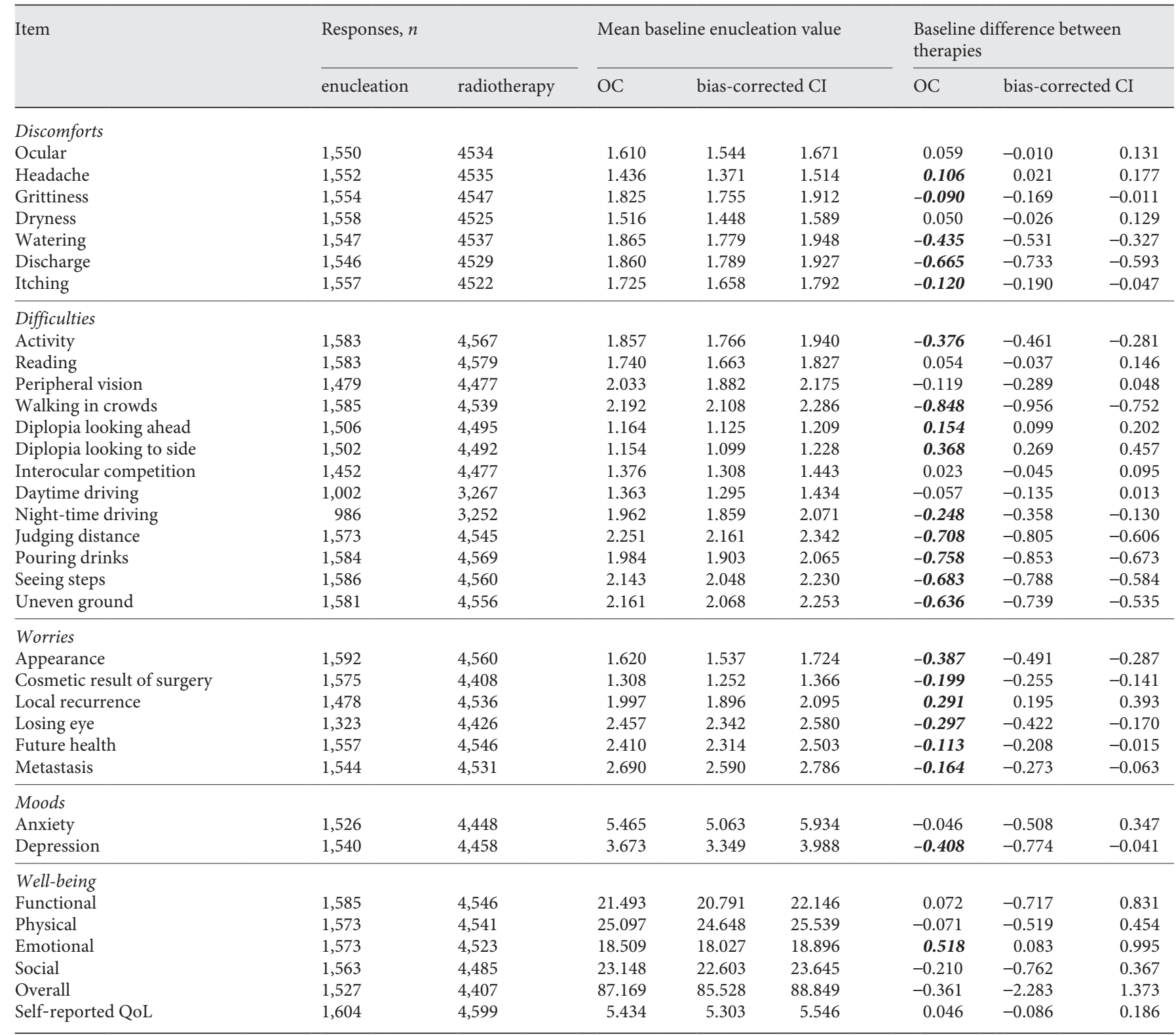

CI, confidence interval; OC, observed coefficient (syn: regression coefficient); QoL, quality of life. Significant differences in bold. When reporting baseline differences in discomforts, difficulties, worries, and moods between therapies, positive OC values indicate worse symptoms after radiotherapy and negative scores indicate worse symptoms after enucleation; however, with well-being and self-reported QoL, positive scores indicate better outcomes after radiotherapy.

\section{Discussion}

\section{Main Findings}

Soon after enucleation, patients experienced visual difficulties because of loss of stereopsis and visual field and were concerned about their appearance and about metastatic disease. After radiotherapy, patients were more concerned about local tumor recurrence and more troubled by diplopia and headache. Over time, visual difficulties diminished after enucleation but increased in pa- 
Table 3 (continued)

b Trends in patient-reported outcome measures, moods, and QoL

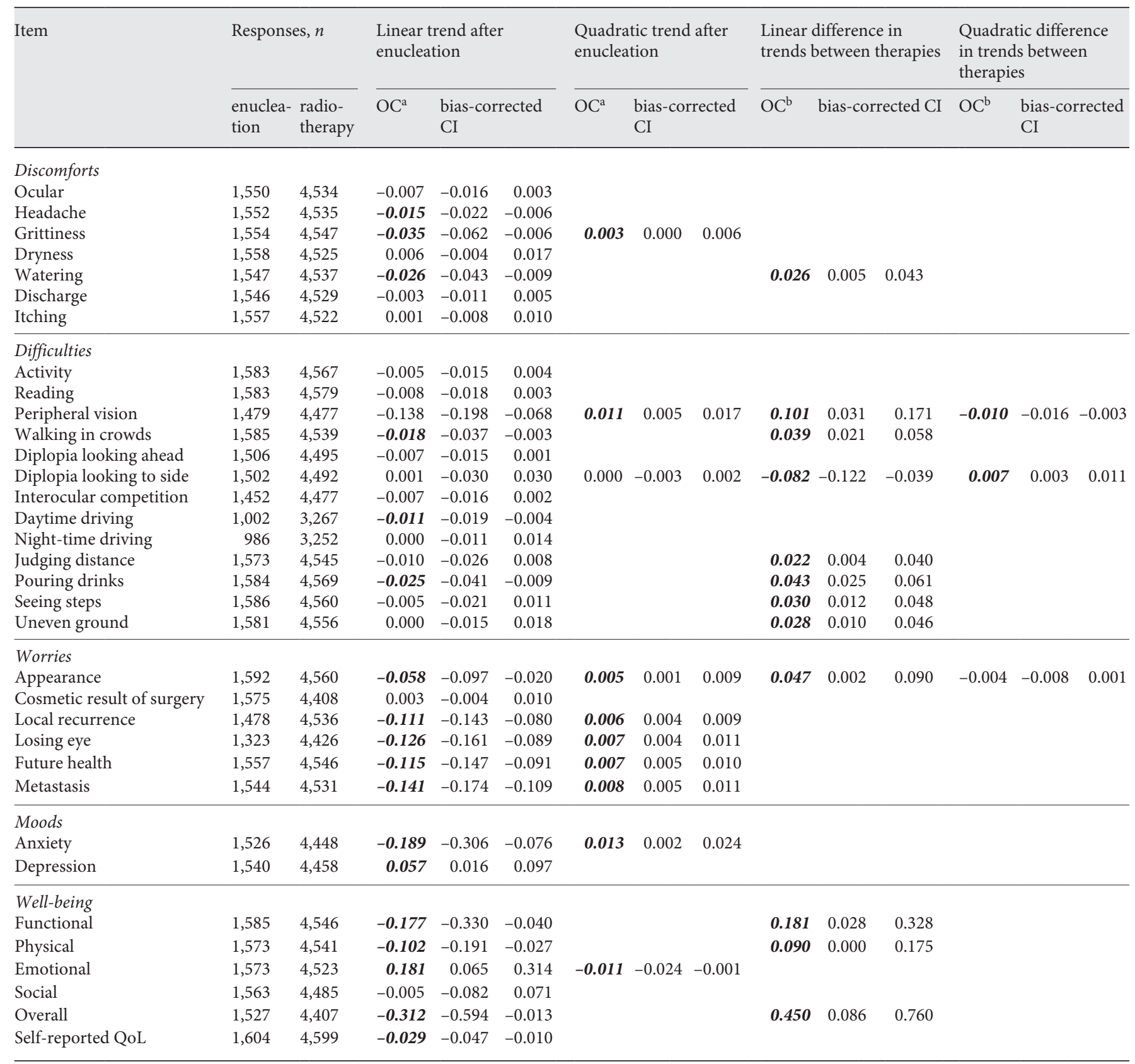

CI, confidence interval; OC, observed coefficient; QoL, quality of life. Significant differences are in bold. ${ }^{\text {a }}$ Observed coefficient represents the approximate change over a time period, with negative values indicating diminishing scores. ${ }^{b}$ Observed coefficient represents the difference between the two groups, for an approximate change over a time period with positive values indicating converging trends. With discomforts, difficulties, worries, and moods, higher values indicate worse outcomes, whereas with well-being and self-reported quality of life, higher values indicate better outcomes.

QoL after Treatment for Choroidal Melanoma
Ocul Oncol Pathol 2019;5:402-411

DOI: $10.1159 / 000496927$ 
tients who had received radiotherapy, with concerns about metastasis, loss of health and tumor recurrence diminishing in both groups. Anxiety tended to diminish whereas depression increased, especially after enucleation. Emotional well-being improved after both kinds of treatment, whereas functional and physical well-being diminished after enucleation but improved after radiotherapy. Self-reported QoL diminished equally with both kinds of treatment.

\section{Results}

We hypothesize that visual function improved after enucleation because patients adjusted to loss of visual field and stereopsis. It is likely that visual difficulties after radiotherapy increased as radiation-induced ocular morbidity developed. Some patients reported diplopia after enucleation, possibly because they confused this with blurred vision or astigmatism.

Enucleated patients were more worried about metastasis; this is probably because they tended to have more advanced and more lethal tumors than those who had received radiotherapy. Understandably, patients who had radiotherapy were more concerned about the possibility of local tumor recurrence than those who had the eye removed. There were a few enucleated patients who reported concerns about local recurrence even though this complication is extremely rare. These fears diminished over time in all patients, and this may explain why emotional well-being improved and why anxiety diminished.

We found that over time there was a decline in selfreported QoL, which occurred in both treatment groups. In our previous study, multivariate analysis showed selfreported QoL to be associated with social support, employment status and self-reported general health but not treatment [6].

Higher levels of depression were found after enucleation, compared to radiotherapy. Multivariable analyses in our previous study showed that depression was not associated with type of treatment but was associated with unemployment, which was more common in enucleated patients [6].

We have compared our results with those of previous studies in our previous report [6]. To our knowledge, only two had a cohort of more than 100 patients. The first investigation, the Collaborative Ocular Melanoma Study (COMS), reported outcomes in 103 patients randomized between radiotherapy and enucleation [1]. Like us, it found that visual difficulties were initially less after radiotherapy than after enucleation although the visual benefits of radiotherapy diminished over time. Unlike our study, the COMS found that concerns about metastasis were greater after radiotherapy, at least until it was reported that ocular conservation was as effective at prolonging life as enucleation. The second investigation was performed by us on a cohort of 261 patients [14]. This showed no difference in any quality-of-life measures between enucleation and radiotherapy, probably because of the small sample size. As in the present study, anxiety diminished with time. Our previous studies indicate that well-being after radiotherapy or enucleation for choroidal melanoma is not significantly worse than that of the general, adult, US population [6].

The main strengths of the study are the large number of patients and the long follow-up. The main weakness is that patients were not randomized between enucleation and radiotherapy; this would have been unethical. In our previous study, we performed multivariate analyses to adjust for baseline differences between the two treatment groups; ocular features were not significant except for monosomy 3 , which was associated with anxiety and reduced emotional well-being [6]. Another weakness is the lack of pre-treatment questionnaires; this was impractical because we treated patients only 1 day after their first visit to our center in Liverpool, unless they felt they needed more time to decide what treatment to have. Further, patients were highly anxious soon after their diagnosis of malignancy so that their questionnaires would not have reflected their usual psychological state. Another limitation is loss to follow-up, which was greater after enucleation because of early discharge from our clinic and because of increased mortality.

Relatively few patients developed serious ocular morbidity after radiotherapy. This is because we avoided this treatment in patients with tumors that were large and/or extending close to optic disc, preferring enucleation or some form of local resection. Our results are therefore not relevant to patients treated with radiotherapy in the presence of adverse clinical features.

Since this study was limited to patients residing in England and Wales, the data may not apply to patients in other countries.

\section{Clinical Implications}

Patients can be informed of the visual difficulties they can expect after enucleation but reassured that these problems are likely to diminish as they adapt to their con-

Fig. 1. Patient-reported outcomes, in terms of discomfort (a), visual difficulties (b), and worries (c) after enucleation or radiotherapy for choroidal melanoma.
(For figure see next page.) 

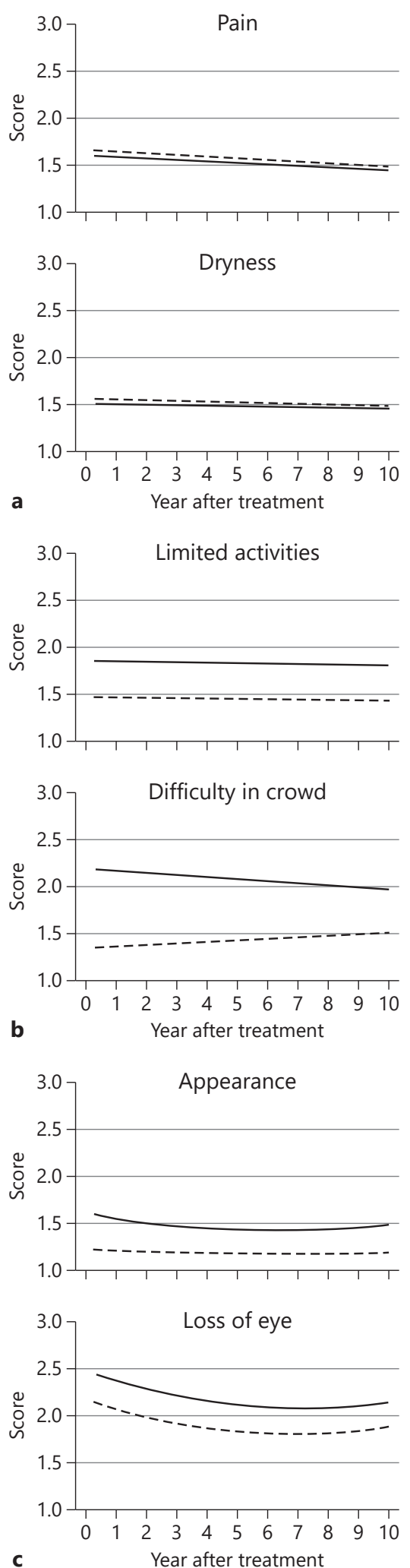
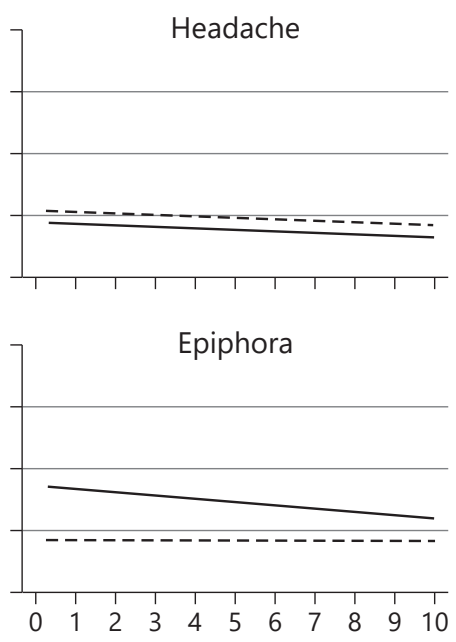

Year after treatment
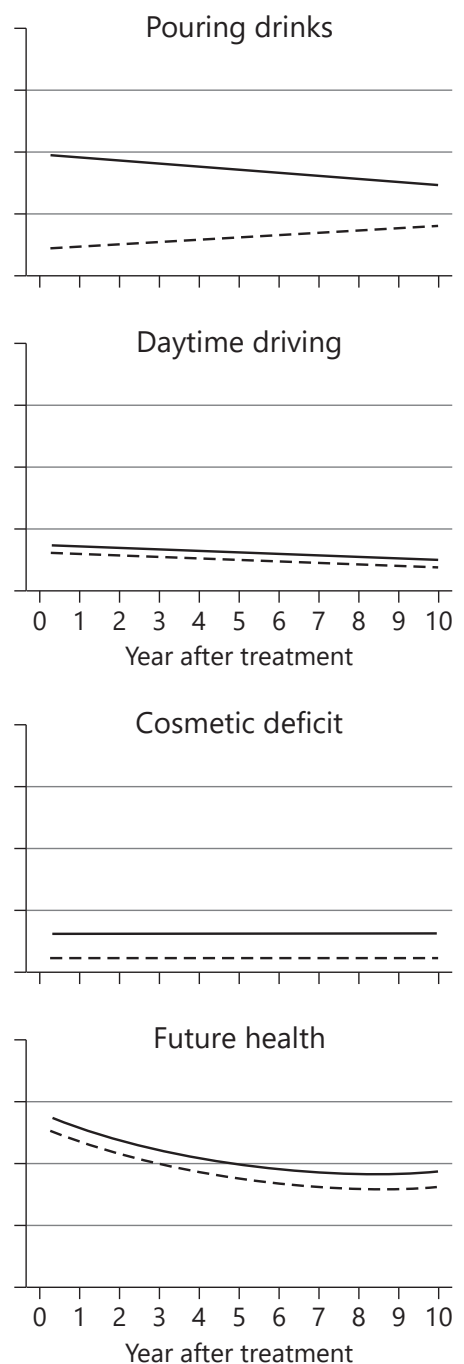

Grittiness

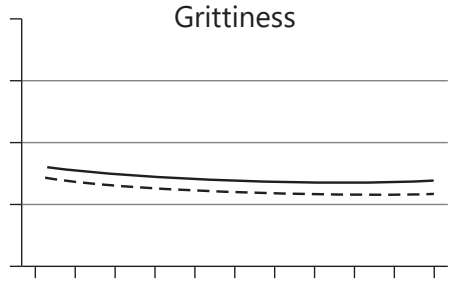

Discharge

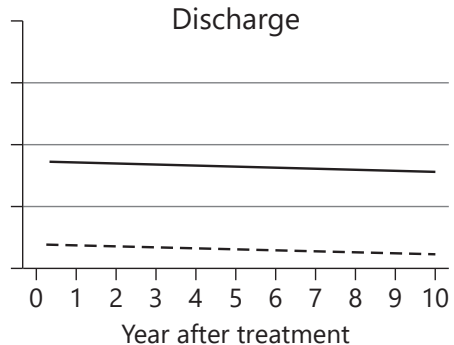

Diplopia looking ahead

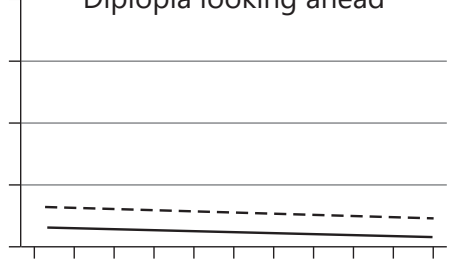

Night-time driving

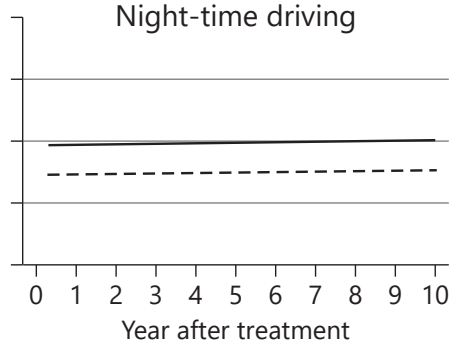

Local recurrence
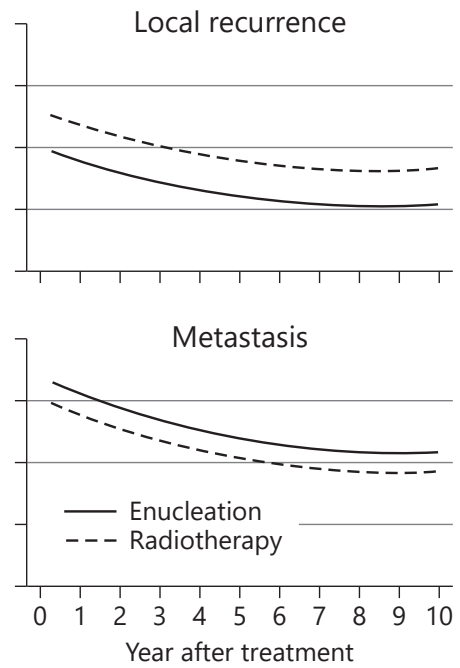
Fig. 2. Moods and QoL after enucleation or
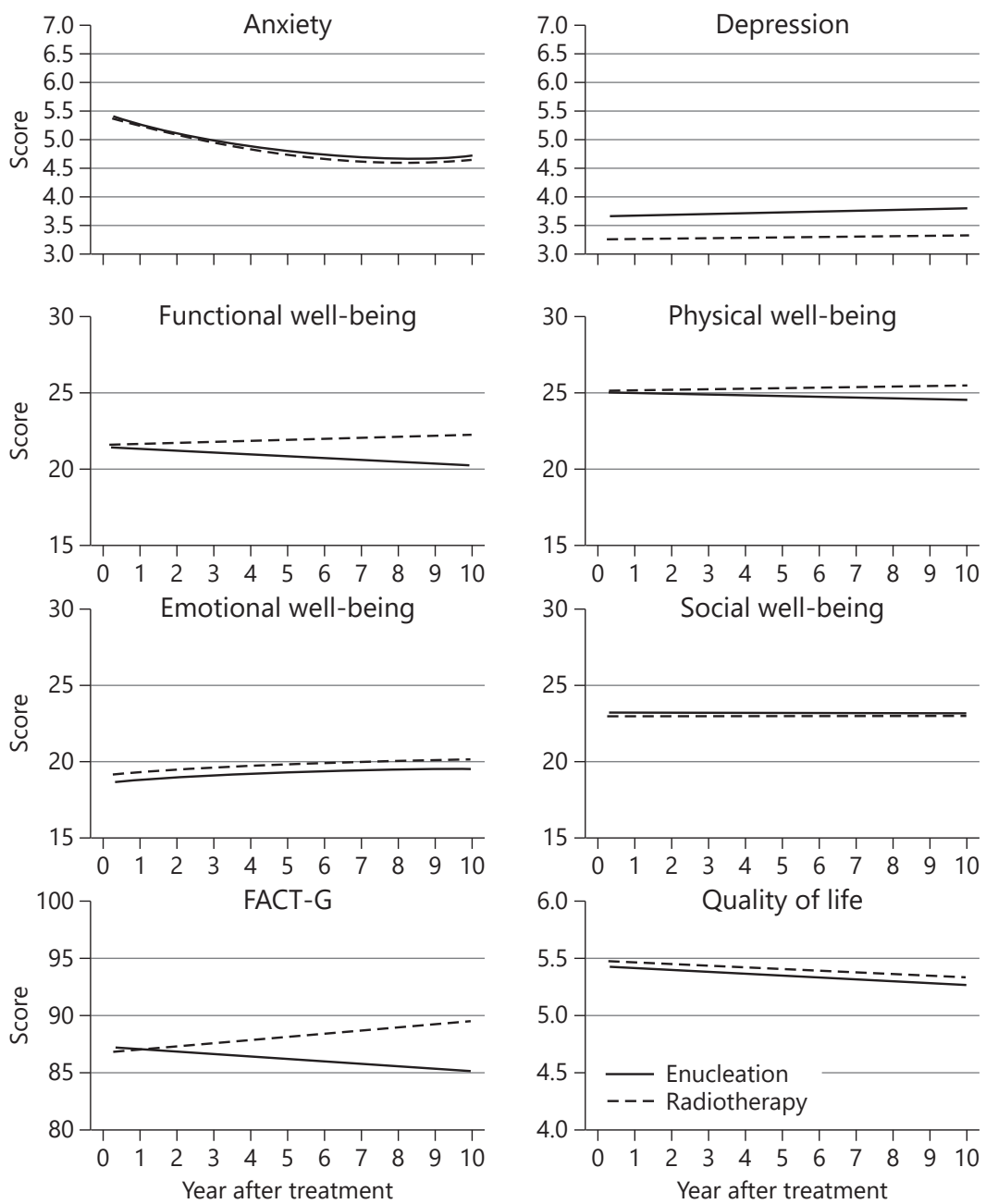
radiotherapy for choroidal melanoma.

dition. Conversely, patients considering radiotherapy should be warned about the way in which ocular morbidity increases over time after this treatment.

An important lesson from this study is that some enucleated patients are concerned about the possibility of local tumor recurrence, even though this complication is exceptionally rare. Similarly, patients with small, disomy-3 melanoma and an excellent prognosis for survival may be unduly concerned about metastatic disease. These results have encouraged us to remind patients about any good prognostic features at every opportunity.

Other encouraging findings from this investigation and our previous study are that symptoms were generally mild. Few patients expressed concerns about their ap- pearance following enucleation, especially once the initial postoperative period was over. Our previous study on this cohort identified factors such as poor social support and poor general health as predictors for anxiety, depression, and poor well-being, thereby making it easier to identify patients who need psychological counseling and other supportive measures [6].

\section{Further Studies}

We found that some of the questions in the EORTCQLQ-OPT30 instrument caused problems. For example, after enucleation, some patients reported diplopia or expressed worries about losing the eye. These findings suggest that the EORTC questionnaire requires further refinement. 
As mentioned, relatively few patients developed problems after radiotherapy, because those with high-risk features tended to have the eye removed. It would be useful to compare QoL after radiotherapy with that after enucleation in patients with large and/or juxtapapillary tumors. We hypothesize that such patients tend to have a better QoL after enucleation.

It would seem useful to develop and evaluate questionnaires aimed at predicting poor QoL after treatment for choroidal melanoma. This would enable special interventions to be targeted at high-risk individuals, such interventions then requiring evaluation.

A survey of 180 patients with uveal melanoma in the United States highlights several needs that patients feel are not being addressed [15]. The most common are lack of psychological support and lack of advice on financial aspects of care. Further studies are indicated to determine how psychological support, counseling, doctor-patient communication, and other interventions influence QoL in patients with uveal melanoma.

\section{Conclusions}

The findings of this study should help physicians understand what patients tend to experience after treatment for choroidal melanoma, according to the type of therapy they receive and the time that has elapsed since this was administered.

\section{Acknowledgements}

We are grateful to Nicola Longrigg and Gary Cheetham of Liverpool Ocular Oncology Centre: St Paul's Eye Unit Royal Liverpool and Broadgreen University Hospitals Trust for their assistance with data management. This work was made possible in part, by NIH-NEI EY002162 - Core Grant for Vision Research and by the Research to Prevent Blindness Unrestricted Grant.

\section{Statement of Ethics}

This project was approved as an audit in 2003 by the Liverpool Research Ethics Committee (03/06/072/A) and was conducted in accordance with the Declaration of Helsinki. The data collection was incorporated into our routine clinical practice and not undertaken as a formal clinical trial.

\section{Disclosure Statement}

The authors declare no conflicts of interest.

\section{References}

1 Melia M, Moy CS, Reynolds SM, Hayman JA, Murray TG, Hovland KR, et al.; Collaborative Ocular Melanoma Study-Quality of Life Study Group. Quality of life after iodine 125 brachytherapy vs enucleation for choroidal melanoma: 5-year results from the Collaborative Ocular Melanoma Study: COMS QOLS Report No. 3. Arch Ophthalmol. 2006 Feb;124(2):226-38.

2 Amaro TA, Yazigi L, Erwenne C. Depression and quality of life during treatment of ocular bulb removal in individuals with uveal melanoma. Eur J Cancer Care (Engl). 2010 Jul; 19(4):476-81.

3 Brandberg Y, Kock E, Oskar K, af Trampe E, Seregard S. Psychological reactions and quality of life in patients with posterior uveal melanoma treated with ruthenium plaque therapy or enucleation: a one year follow-up study. Eye (Lond). 2000 Dec;14(Pt 6):839-46.

4 Chabert S, Velikay-Parel M, Zehetmayer M. Influence of uveal melanoma therapy on patients' quality of life: a psychological study. Acta Ophthalmol Scand. $2004 \mathrm{Feb} ; 82(1): 25-31$.

5 Cruickshanks KJ, Fryback DG, Nondahl DM, Robinson N, Keesey U, Dalton DS, et al. Treatment choice and quality of life in patients with choroidal melanoma. Arch Ophthalmol. 1999 Apr;117(4):461-7.
6 Damato B, Hope-Stone L, Cooper B, Brown SL, Salmon P, Heimann H, et al. Patient-reported Outcomes and Quality of Life After Treatment of Choroidal Melanoma: A Comparison of Enucleation Versus Radiotherapy in $1596 \mathrm{~Pa}-$ tients. Am J Ophthalmol. 2018 Sep;193:230-51.

7 Damato B, Eleuteri A, Taktak AF, Coupland SE. Estimating prognosis for survival after treatment of choroidal melanoma. Prog Retin Eye Res. 2011 Sep;30(5):285-95.

8 Ah-Fat FG, Sharma MC, Damato BE. Taping outpatient consultations: a survey of attitudes and responses of adult patients with ocular malignancy. Eye (Lond). 1998;12(Pt 5):789-91.

9 Brandberg Y, Damato B, Kivelä T, Kock E, Seregard S; EORTC Ophthalmic Oncology Task Force; EORTC Quality of Life Group. The EORTC ophthalmic oncology quality of life questionnaire module (EORTC QLQOPT30). Development and pre-testing (Phase I-III). Eye (Lond). 2004 Mar;18(3):283-9.

10 Zigmond AS, Snaith RP. The hospital anxiety and depression scale. Acta Psychiatr Scand. 1983 Jun;67(6):361-70.
11 Cella DF, Tulsky DS, Gray G, Sarafian B, Linn E, Bonomi A, et al. The Functional Assessment of Cancer Therapy scale: development and validation of the general measure. J Clin Oncol. 1993 Mar;11(3):570-9.

12 Brucker PS, Yost K, Cashy J, Webster K, Cella D. General population and cancer patient norms for the Functional Assessment of Cancer Therapy-General (FACT-G). Eval Health Prof. 2005 Jun;28(2):192-211.

13 Pearman T, Yanez B, Peipert J, Wortman K, Beaumont J, Cella D. Ambulatory cancer and US general population reference values and cutoff scores for the functional assessment of cancer therapy. Cancer. 2014 Sep;120(18): 2902-9.

14 Hope-Stone L, Brown SL, Heimann H, Damato B, Salmon P. Two-year patient-reported outcomes following treatment of uveal melanoma. Eye (Lond). 2016 Dec;30(12):1598605.

15 Afshar AR, Deiner M, Allen G, Damato BE. The Patient's Experience of Ocular Melanoma in the US: A Survey of the Ocular Melanoma Foundation. Ocul Oncol Pathol. 2018 Sep;4(5):280-90. 